\title{
ORIGINAL
}

\section{INCIDENCIA DE CARDIOPATÍA ISQUÉMICA Y ACCIDENTE CEREBROVASCULAR EN TRABAJADORES DE UNA ADMINISTRACIÓN LOCAL DEL SUR DE ESPAÑA} A LO LARGO DE DIEZ AÑOS DE SEGUIMIENTO

\author{
Carlos Álvarez-Fernández (1), Manuel Romero-Saldaña (1), Carlos Álvarez-López (2) y Manuel Vaquero-Abellán (3) \\ (1) Departamento de Seguridad y Salud Laboral. Ayuntamiento de Córdoba. Córdoba. España. \\ (2) Distrito Sanitario Guadalquivir (Córdoba). Servicio Andaluz de Salud. Córdoba. España. \\ (3) Dirección General de Prevención y Protección Ambiental. Universidad de Córdoba. Córdoba. España.
}

Los autores declaran que no existe conflicto de intereses.

\section{RESUMEN}

Fundamentos: Las enfermedades cardiovasculares son la primera causa de muerte en España. Existen escasos estudios sobre incidencia de acontecimientos cardiovasculares en trabajadores. Los objetivos de este trabajo fueron conocer la incidencia de cardiopatía isquémica (CI) y enfermedad cerebrovascular (ECV) en trabajadores de una administración pública del sur de España, estimar la prevalencia de los factores de riesgo cardiovascular (FRCV) y su contribución a la misma, así como evaluar el comportamiento predictivo de la función REGICOR.

Métodos: Se realizó un estudio longitudinal de cohortes históricas, no concurrentes, constituidas por 698 trabajadores, reclutados en los exámenes de salud laboral. Las variables dependientes fueron la aparición de CI o ECV, mientras que las principales independientes fueron glucosa, colesterol, triglicéridos, Índice de Masa Corporal (IMC), presión arterial, perímetro abdominal, actividad física, tabaquismo y puntuación REGICOR. Se llevó a cabo una regresión de Cox y se calculó el área $\mathrm{ABC}$ de la curva ROC para las variables explicativas.

Resultados: Según la función REGICOR, se clasificó de alto riesgo al $0,6 \%$ de la población. La Densidad de Incidencia (DI) por cada 100.000 personas-año para cardiopatía isquémica en mujeres resultó ser de 53,9 por 357,4 en hombres, sin diferencias significativas respecto a las esperadas a partir de la función REGICOR. La DI combinada para cardiopatía isquémica y accidentes cerebrovasculares fue de 477,1 por cada 100.000 personas-año (en hombres).

Conclusiones: La función REGICOR valora adecuadamente el riesgo cardiovascular de manera global, perdiendo capacidad predictiva según grupos de riesgo. El tabaquismo y la presión arterial se muestran como los factores de riesgo modificables con mayor asociación independiente en la aparición de una enfermedad cardiovascular.

Palabras clave: Enfermedad coronaria, Accidente cerebrovascular, Incidencia. Trabajadores, Factores de riesgo, Funciones de riesgo, Síndrome metabólico.

Correspondencia:

Carlos Álvarez Fernández

C/ Palacio de la Galiana, 19

14012 Córdoba, España

craf.19arauco@hotmail.com

\section{ABSTRACT \\ Incidence of ischemic heart disease and stroke in workers of a local administration in southern Spain over ten years of follow-up}

Background: Cardiovascular disease is the first cause of death in Spain. There are a few studies about the incidence of ischemic heart disease and cerebrovascular disease in workers. The objectives of this study were to determine the incidence of coronary and cerebrovascular disease in a cohort of workers of a public administration in the south of Spain, to estimate the prevalence of CVRF and its contribution to it, as well as to evaluate the predictive behavior of the REGICOR function.

Methods: Longitudinal historical cohort study, not concurrent, consisting of 698 workers, recruited from occupational health examinations. The dependent variables were the appearance of $\mathrm{CI}$ or CVD while leading independent were glucose, cholesterol, triglycerides, BMI, blood pressure, waist circumference, physical activity, smoking and REGICOR score and Score. Is performed Cox regression and its calculated area AUC of the ROC curve area for the explanatory variables.

Results: According to the REGICOR function, $0.6 \%$ of the population was classified as high risk. The Incidence Density per 100,000 persons-year for ischemic heart disease in women was found to be 53.9 by 357.4 in men, without significant differences with respect to those expected from the REGICOR function. The combined DI for ischemic heart disease and stroke was 477.1 per 100,000 person-years (men).

Conclusions: The REGICOR function adequately assesses cardiovascular risk globally, losing predictive capacity according to risk groups. Smoking and blood pressure are shown as modifiable risk factors with greater independent association in the onset of cardiovascular disease.

Key words: Coronary heart disease, Cerebrovascular diseases, Incidence, Workers, Risk factor's, Risk functions, Metabolic syndrome.

Cita sugerida: Álvarez-Fernández C, Romero-Saldaña M, Álvarez-López C y Vaquero-Abellán M. Incidencia de cardiopatía isquémica y accidente cerebrovascular en trabajadores de una administración local del sur de España a lo largo de diez años de seguimiento. Rev Esp Salud Pública. 2019;94: 7 de enero e201001001. 


\section{INTRODUCCIÓN}

En España, según el Instituto Nacional de Estadística $^{(1)}$, el 28,8\% de las defunciones de 2017 tuvieron a las enfermedades circulatorias como causa básica de mortalidad. Estas, con 263,3 muertes por cada 100.000 habitantes, siguieron siendo la primera causa de mortalidad global, primera también en mujeres $(279,9$ en el mismo rango) y segunda en hombres (246,1 en el mismo rango) tras los tumores. De los 122.466 fallecimientos a causa de enfermedades circulatorias, el 48,4\% fueron secundarias por cardiopatía isquémica $(\mathrm{CI})$ y accidente cerebrovascular (ACV).

La tendencia descendente en la mortalidad cardiovascular observada en las últimas décadas ${ }^{(2,3,4)}$ parece deberse al efecto de la prevención primaria sobre el control de los factores de riesgo cardiovascular (FRCV), así como a las intervenciones terapéuticas en fase aguda y prevención secundaria ${ }^{(5)}$.

Los trabajos sobre incidencia de CI y ACV en España son escasos, abordando aspectos diferentes de las mismas y dificultando su conocimiento fiable $\mathrm{e}^{(6,7,8,9,10,11,12)}$. Tras la cohorte de Manresa $(1968 / 96)^{(13)}$, son pocos los trabajos sobre incidencia relativos a la población laboral posteriores al año 2000. Esta escasez influye en que la capacidad de las diversas ecuaciones de cálculo del riesgo cardiovascular para identificar a los trabajadores que desarrollarán eventos cardiovasculares sea poco conocida ${ }^{(14)}$.

Las actividades preventivas implican priorización y, para ello, se precisa conocer el impacto de cada posible intervención en el riesgo de la población. La prevención primaria de las enfermedades cardiovasculares se fundamenta en el diagnóstico y control de los factores de riesgo cardiovascular (FRCV) mediante una intervención multifactorial. En España, la información que se tiene sobre el riesgo coronario atribuible a cada factor de riesgo ${ }^{(15,16)}$ es insuficiente.

Los objetivos de este trabajo fueron conocer la incidencia de cardiopatía isquémica y enfermedad cerebrovascular en trabajadores de una administración pública, estimar la prevalencia de los FRCV y su contribución a la misma, así como evaluar el comportamiento predictivo de la función REGICOR.

\section{SUJETOS Y MÉTODOS}

Se llevó a cabo un estudio longitudinal de cohortes históricas, no concurrente, durante el periodo 2007-2017. A partir de los datos de incidencia de eventos cardiovasculares que el estudio Dieta y Riesgo de Enfermedad Cardiovascular España ${ }^{(17)}$ (DRECE II) presenta para grupos de alto riesgo $(690 \times 100.000)$ y bajo riesgo (172 x 100.000), y mediante el uso del programa EPIDAT ver. 4.1, se calculó el tamaño para un estudio de cohortes con una potencia mínima del $80 \%$ y nivel de confianza del $95 \%$, considerando la posibilidad de pérdidas de sujetos y una razón de no expuestos/expuestos de 0,6 , resultando una muestra de 6.800 personas/año.

La población diana estuvo constituida por la totalidad de los trabajadores (1.158) pertenecientes a la plantilla del Ayuntamiento de Córdoba con contrato indefinido cuya edad se encontraba entre los 35 y los 60 años al inicio del estudio. Fueron excluidas 115 personas por presentar antes del inicio del estudio cardiopatía isquémica, accidente cerebrovascular, arteriopatía periférica, insuficiencia cardiaca, haber sido trasladado de puesto de trabajo por razón de salud, haber presentado al menos un periodo de incapacidad temporal superior a un año o encontrarse en proceso de incapacitación laboral permanente, siendo finalmente 1.043 los sujetos a estudio. Negaron su participación 247 y se perdieron durante el estudio 98 , con lo que 
la población constó de 698 trabajadores reclutados en la vigilancia de la salud realizada por la unidad de salud laboral de la administración. Los reconocimientos médicos fueron de carácter voluntario. De los que no participaron se conservaron datos de edad y sexo, no presentando diferencias significativas respecto de los que fueron objeto del estudio. El tiempo medio de seguimiento fue de 9,8 años por persona.

Variables, medidas y análisis de los datos. Se determinó como variable de exposición la propia calificación de la función REGICOR, de tal forma que fueron considerados "No expuestos" todos aquellos trabajadores cuya puntuación les incluía en el grupo de bajo riesgo, y como "Expuestos" a los grupos de riesgo moderado, alto y muy alto.

La variable resultado fue padecer o no evento de CI (presentar primer episodio de angina de pecho o infarto agudo de miocardio, fatal o no).

Variables independientes:

- Variables de persona y estilos de vida: edad, sexo, tabaquismo, consumo de alcohol ${ }^{(18)}$, actividad física ${ }^{(19)}$, años de formación académica y puesto de trabajo (administración, policía, bombero, oficios manuales, capataces, conductores, ordenanzas).

- Variables analíticas: colesterol total en mg/ dl (CT), colesterol unido a lipoproteínas de baja densidad en mg/dl (cLDL), colesterol unido a lipoproteínas de alta densidad en mg/dl (cHDL), triglicéridos en $\mathrm{mg} / \mathrm{dl}$ (TG), ácido úrico en $\mathrm{mg} /$ dl (AU), glucosa basal en mg/dl (GLUC), índices aterogénicos de Castelli (CT/cHDL), índice de Kannel (cLDL/cHDL) y TG/cHDL.

- Variables antropométricas: peso, talla, índice de masa corporal (IMC), circunferencia de cintura (CC), índice de cintura/talla (ICT), presión arterial sistólica y diastólica $(\mathrm{mmHg})$, presión diferencial de pulso, síndrome metabólico $^{(20)}$, riesgo coronario según algoritmo derivado del Registro Gironí del Cor (REGICOR) (21), riesgo de mortalidad por evento cardiovascular según Score calibrado para España ${ }^{(22)}$ y porcentaje de peso graso $(\% \mathrm{pg})$ según modelo de Deurenberg; (hombre=1 y mujer=0). Se empleó la siguiente fórmula:

$$
\begin{gathered}
\% \mathrm{Pg}=1,2 \times(\mathrm{IMC})+0,23 \times(\text { edad en años }) \\
-10,8 \times(\text { sexo })-5,4 .
\end{gathered}
$$

Para talla y peso se utilizó báscula y estadiómetro Atlántida S11. La circunferencia de cintura fue medida mediante cinta metálica flexible con gradación en milímetros (Cescorf), siguiendo para las variables antropométricas las normas de la International Society for the Advancement of Kinanthropometry (ISAK) ${ }^{(23)}$. La presión arterial se midió mediante esfingomanómetro automático (OMROM-M3) y según normas de la Guía de práctica clínica de la ESH/ESC para el manejo de la Hipertensión arterial-2013 ${ }^{(24)}$. Las muestras de sangre se obtuvieron mediante venopunción tras doce horas de ayuno, siendo analizadas siguiendo procedimientos normalizados automatizados en bioquímica clínica (autoanalizador ILAB-600).

Las variables analizadas se midieron al inicio de la cohorte. Peso, talla, perímetro abdominal, medida de la presión arterial y venopunción para analitos son técnicas realizadas por un solo profesional enfermero.

Análisis estadístico. Las variables cuantitativas se presentaron por su media y desviación estándar, mientras que las variables cualitativas se mostraron en forma de porcentajes. En el caso de la variable resultado se determinó la densidad de incidencia, riesgo relativo, riesgo atribuible y fracción atribuible del riesgo.

Con el fin de comprobar la bondad de ajuste a una distribución normal de variables cuantitativas 
continuas o discretas, se empleó la prueba de Kolmogorov-Smirnov si $\mathrm{N}>50$, o la prueba de Shapiro-Wilk si $\mathrm{N}<50$, según fue indicado. Para contrastar las diferencias entre dos medias independientes, se utilizó la prueba t de Student o U de Mann-Whitney, según procedía. La comparación de porcentajes se realizó mediante el test chi-cuadrado, aplicando el test exacto de Fisher cuando al menos el $20 \%$ de las frecuencias esperadas era inferior a $5 \mathrm{y}$ ninguna frecuencia esperada inferior a 1. Se determinó el área bajo la curva ROC para conocer la capacidad predictiva de las variables explicativas.

Se realizó una regresión Cox en la que se calculó tanto el modelo ajustado como no ajustado, determinando en cada caso los valores de Hazard Ratio para cada variable independiente.
Para la comprobación de la condición de riesgos proporcionales se aplicó el método analítico de Hosmer-Lemeshow. En todos los casos, el intervalo de confianza se calculó con una seguridad del 95\%.

Bioética. El consentimiento informado se obtuvo conforme a la legislación vigente. El protocolo de estudio cumplía con la Declaración de Helsinki para estudios médicos y fue aprobado por la comisión de bioética del programa de doctorado de la Universidad de Córdoba.

\section{RESULTADOS}

La población estudiada presentó un 73,3\% de hombres. Policía, bomberos y oficios manuales presentaron una mayor proporción de

\begin{tabular}{|c|c|c|c|c|c|c|}
\hline \multicolumn{7}{|c|}{$\begin{array}{c}\text { Tabla } 1 \\
\text { Comparación entre trabajadores que abandonan el estudio y trabajadores que finalizan. }\end{array}$} \\
\hline \multirow{2}{*}{ Variables } & \multicolumn{2}{|c|}{ Mujeres (30) } & \multicolumn{2}{|c|}{ Hombres (68) } & \multirow{2}{*}{$\frac{\text { Mujeres }}{\mathrm{p}^{(*)}}$} & \multirow{2}{*}{$\frac{\text { Hombres }}{\mathrm{p}^{(*)}}$} \\
\hline & $\bar{X}$ & $\mathrm{DE}$ & $\bar{X}$ & $\mathrm{DE}$ & & \\
\hline CT (mg/dl) & 203,5 & 41,5 & 210,5 & 33,9 & 0,85 & 0,69 \\
\hline cHDL (mg/dl) & 62,0 & 15,3 & 54,5 & 11,0 & 0,76 & 0,58 \\
\hline cLDL (mg/dl) & 120,7 & 35,1 & 133,3 & 31,1 & 0,96 & 0,66 \\
\hline GLUC (mg/dl) & 95,6 & 29,2 & 100,6 & 25,8 & 0,55 & 0,09 \\
\hline TG (mg/dl) & 86,1 & 55,1 & 131,2 & 85,3 & 0,77 & 0,64 \\
\hline IMC & 24,0 & 3,5 & 27,4 & 3,7 & 0,12 & 0,83 \\
\hline PAS (mmHg) & 114 & 18,3 & 124,1 & 10,4 & 0,63 & 0,72 \\
\hline PAD (mmHg) & 73,8 & 7,7 & 78,5 & 7,7 & 0,94 & 0,09 \\
\hline $\mathrm{CC}(\mathrm{cm})$ & 77,6 & 9,4 & 93,1 & 10,2 & 0,67 & 0,88 \\
\hline Regicor rcv (\%) & 1,9 & 1,7 & 2,9 & 1,7 & 0,76 & 0,65 \\
\hline Score rev (\%) & 0,2 & 0,4 & 1 & 0,9 & 0,19 & 0,39 \\
\hline Edad (años) & 43,2 & 5,8 & 43,5 & 5,3 & 0,86 & 0,38 \\
\hline \multirow{2}{*}{ Variables } & \multicolumn{2}{|c|}{ Mujeres } & \multicolumn{2}{|c|}{ Hombres } & Mujeres & Hombres \\
\hline & $\%$ & Ic95\% & $\%$ & Ic95\% & $\mathrm{p}^{(* *)}$ & $\mathrm{p}^{(* *)}$ \\
\hline Tabaco & 36,7 & $(19,9-56,1)$ & 37,5 & $(25,-49,3)$ & 0,79 & 0,87 \\
\hline $\mathbf{A F B}^{(* * *)}$ & 36,3 & $(19,9-56,1)$ & 22,5 & $(12,4-33,7)$ & 0,85 & 0,75 \\
\hline \multicolumn{7}{|c|}{$\begin{array}{l}\text { CT: colesterol total; cHDL: colesterol unido a lipoproteínas de alta densidad; cLDL: colesterol } \\
\text { unido a lipoproteínas de baja densidad; GLUC: glucosa; TG: triglicéridos; IMC: índice de masa } \\
\text { corporal; PAS: presión arterial sistólica; PAD: presión arterial diastólica; CC: circunferencia } \\
\text { abdominal; REGICOR: función de riesgo cardiovascular según registro gironí del corazón; } \\
(*) \text { p: comparación medias entre los participantes del estudio y los que lo a abandonan; } \\
(* *) \text { p: comparación proporciones entre participantes del estudio y los que lo abandonan; } \\
(* * *) \text { AFB: según cuestionario internacional actividad física IPAQ. }\end{array}$} \\
\hline
\end{tabular}


hombres, mientras que las mujeres fueron mayoría en puestos de administración. No hubo diferencias en el grupo de ordenanzas $(p=0,54)$.

La tabla 1 compara las características de los trabajadores que abandonaron el estudio frente a aquellos que lo finalizaron (variables analíticas, antropométricas, estilos de vida, etc.), no existiendo diferencias significativas entre ambos grupos.

La tabla 2 muestra las medias de las variables analíticas y antropométricas de la muestra según

\begin{tabular}{|c|c|c|c|c|c|c|c|}
\hline \multicolumn{8}{|c|}{$\begin{array}{c}\text { Tabla } 2 \\
\text { Características de la muestra de estudio. Prevalencia de principales factores } \\
\text { de riesgo cardiovascular. FRCV. Variables analíticas y antropométricas } \\
\text { y prevalencia de factores de riesgo cardiovascular. }\end{array}$} \\
\hline \multirow{2}{*}{ Variables } & \multicolumn{2}{|c|}{ Mujeres } & \multicolumn{2}{|c|}{ Hombres } & \multirow[b]{2}{*}{$\mathrm{p}$} & \multicolumn{2}{|c|}{ Global } \\
\hline & $\bar{x}$ & $\mathrm{DE}$ & $\bar{x}$ & $\mathrm{DE}$ & & $\bar{x}$ & $\mathrm{DE}$ \\
\hline CT (mg/dl) & 200,2 & 34,4 & 214,3 & 37,3 & $<0,001$ & 210,7 & 37,1 \\
\hline cHDL (mg/dl) & 60,3 & 13,5 & 53,7 & 11,5 & $<0,001$ & 55,4 & 12,4 \\
\hline c LDL(mg/dl) & 121,0 & 30,3 & 135,1 & 33,6 & $<0,001$ & 131,4 & 33,3 \\
\hline GLUC (mg/dl) & 92,7 & 17,1 & 99,0 & 16,8 & $<0,001$ & 97,4 & 17,1 \\
\hline $\mathbf{A U}(\mathbf{m g} / \mathbf{d l})$ & 3,4 & 1,1 & 5,7 & 4,5 & $<0,001$ & 5,1 & 4,03 \\
\hline TG (mg/dl) & 82,9 & 48,1 & 126,1 & 80,6 & $<0,001$ & 114,8 & 75,9 \\
\hline IMC & 25,1 & 4,4 & 27,3 & 3,8 & $<0,001$ & 26,8 & 4,08 \\
\hline PAS (mmHg) & 115,7 & 15,2 & 124,6 & 13,1 & $<0,001$ & 122,3 & 14,2 \\
\hline PAD (mmHg) & 73,9 & 8,7 & 80,2 & 8,5 & $<0,001$ & 78,5 & 9,0 \\
\hline PDP (mmHg) & 41,6 & 9,5 & 44,5 & 8,9 & $<0,001$ & 44,8 & 9,2 \\
\hline $\mathrm{CC}(\mathrm{cm})$ & 78,4 & 10,3 & 93,3 & 9,1 & $<0,001$ & 89,4 & 11,5 \\
\hline ICT & 0,49 & 0,07 & 0,54 & 0,05 & $<0,001$ & 0,53 & 0,06 \\
\hline $\operatorname{Pg}(\%)$ & 34,7 & 5,9 & 26,8 & 4,9 & $<0,001$ & 28,8 & 6,2 \\
\hline CT/cHDL & 3,5 & 0,9 & 4,1 & 0,8 & $<0,001$ & 3,9 & 0,9 \\
\hline cLDL/cHDL & 2,1 & 0,7 & 2,6 & 0,7 & $<0,001$ & 2,5 & 0,7 \\
\hline TG/cHDL & 1,5 & 1,1 & 2,4 & 1,6 & $<0,001$ & 2,2 & 1,6 \\
\hline Regicor rev (\%) & 1,8 & 1,4 & 3,0 & 1,8 & $<0,001$ & 2,7 & 1,7 \\
\hline Edad (años) & 43,0 & 5,34 & 44,2 & 5,6 & 0,01 & 43,8 & 5,6 \\
\hline Prevalencia FRCV & \multicolumn{2}{|c|}{ Mujeres } & \multicolumn{2}{|c|}{ Hombres } & \multicolumn{2}{|c|}{ Global } & $\mathrm{p}$ \\
\hline Tabaco & \multicolumn{2}{|c|}{39,3} & \multicolumn{2}{|c|}{35,7} & \multicolumn{2}{|c|}{36,6} & 0,33 \\
\hline $\mathbf{A F B}^{(\mathbf{a})}$ & \multirow{2}{*}{\multicolumn{2}{|c|}{34,9}} & & & $<0,001$ \\
\hline HTA $^{(\mathbf{b})}$ & 11,5 & & \multirow{2}{*}{\multicolumn{2}{|c|}{$\begin{array}{l}22,8 \\
47,0\end{array}$}} & & & $<0,001$ \\
\hline HiperCT $^{(\mathbf{c})}$ & \multicolumn{2}{|c|}{35,1} & & & \multicolumn{2}{|c|}{$\frac{20,0}{43,8}$} & $<0,001$ \\
\hline Obesidad $^{(\mathrm{d})}$ & \multirow{2}{*}{\multicolumn{2}{|c|}{$\frac{13,1}{3,3}$}} & \multicolumn{2}{|c|}{18,2} & \multicolumn{2}{|c|}{16,9} & $<0,001$ \\
\hline Diabetes $^{(\mathrm{e})}$ & & & \multicolumn{2}{|c|}{4,9} & \multicolumn{2}{|c|}{4,4} & $<0,001$ \\
\hline Smet $^{(f)}$ & \multicolumn{2}{|c|}{10,2} & \multicolumn{2}{|c|}{18,8} & \multicolumn{2}{|c|}{16,5} & 0,35 \\
\hline \multicolumn{8}{|c|}{$\begin{array}{l}\text { CT: colesterol total; cHDL: colesterol unido a lipoproteínas de alta densidad; cLDL: colesterol } \\
\text { unido a lipoproteínas de baja densidad; GLUC: glucosa; TG: triglicéridos; IMC: índice de masa } \\
\text { corporal; PAS: presión arterial sistólica; PAD: presión arterial diastólica; CC: circunferencia } \\
\text { abdominal; REGICOR: función de riesgo cardiovascular según registro gironí del corazón; (a) } \\
\text { Actividad física baja: según cuestionario internacional actividad física IPAQ; (b) HTA: Guía de } \\
\text { práctica clínica de la ESH/ESC para el manejo de la hipertensión Arterial, 2013; (c) Hipercoles- } \\
\text { terolemia. PAPPS. Actualización 2014; (d) Obesidad: criterios de la Sociedad Española para el } \\
\text { estudio de la Obesidad. Consenso 2007; (e) Diabetes: criterios Asociación Americana de Diabe- } \\
\text { tes 2015; (f) SMet: Síndrome metabólico (SMet) según criterios de la definición armonizada. }\end{array}$} \\
\hline
\end{tabular}


sexo y la prevalencia de factores de riesgo cardiovascular. Las correspondientes a las variables de CT e IMC señalaron hipercolesterolemia y sobrepeso. El riesgo global calculado de CI a diez años fue de 2,7 (DE 1,7), siendo de 1,7 a 1 en hombres respecto a mujeres. Se observaron diferencias significativas en todos los FRCV analizados entre hombres y mujeres, excepto en tabaquismo y diabetes.

Del total de 698 trabajadores, 77 (11\%) fueron expuestos, siendo el $13,7 \%$ de los hombres (70) y el 3,8\% (7) de las mujeres, con diferencias según género. Se clasificó según REGICOR de alto riesgo al $0,6 \%$ de la población, sin diferencias por sexo $(\mathrm{p}=0,94)$, así como al $10,5 \%$ como moderado y el $88,9 \%$ como bajo, existiendo diferencias por género, especialmente en el grupo de riesgo moderado (13,1\% de hombres frente a 3,2\% de mujeres) (tabla 3 ).

La densidad de incidencia para los eventos coronarios (19 casos) resultó ser de 357,4 por cada 100.000 personas-año en hombres y 276,3 por cada 100.000 personas-año en población total, con una razón de tasas entre no expuestos/expuestos de 0,094 y una fracción atribuible en expuestos de 0,92 (tabla 4).

La tabla 5 muestra los casos y densidad de incidencia esperada (según Regicor) y los casos y densidad de incidencia observados.

\begin{tabular}{|c|c|c|c|c|c|}
\hline \multicolumn{7}{|c|}{ Tabla 3 } \\
Distribución grupos de riesgo REGICOR y exposición según género. \\
\hline Variables & Bajo (\%) & Moderado (\%) & Alto (\%) & Expuestos (\%) & No expuestos (\%) \\
\hline Hombres & 86,3 & 13,1 & 0,59 & 13,7 & 86,3 \\
\hline Mujeres & 96,2 & 3,2 & 0,54 & 3,8 & 96,2 \\
\hline Global & 88,9 & 10,5 & 0,57 & 11,0 & 89,0 \\
\hline p & $<0,001$ & $<0,001$ & 0,94 & \multicolumn{2}{c|}{$<0,001$} \\
\hline
\end{tabular}

\section{Tabla 4}

Densidad de incidencia, razón de tasas incidencia, riesgo atribuible y fracción atribuible en expuestos según ecuaciones REGICOR.

\begin{tabular}{|c|c|c|c|c|c|c|c|c|}
\hline \multirow{2}{*}{\multicolumn{2}{|c|}{ Variables }} & \multicolumn{3}{|c|}{ Densidad de incidencia $^{(a)}$} & \multirow{2}{*}{$\begin{array}{c}\text { Razón } \\
\text { de } \\
\text { tasas }\end{array}$} & \multirow{2}{*}{ Ic95\% } & \multirow{2}{*}{$\operatorname{Rae}^{(d)}$} & \multirow{2}{*}{$\mathrm{FAe}^{(\mathrm{e})}$} \\
\hline & & No expuestos ${ }^{(b)}$ & Expuestos $^{(\mathrm{c})}$ & Global & & & & \\
\hline \multirow{3}{*}{ REGICOR } & Hombres & 159,6 & $1.705,4$ & 357,4 & 0,094 & $0,031-0,264$ & $1.545,8$ & 0,91 \\
\hline & Mujeres & 56,0 & - & 53,9 & - & - & - & - \\
\hline & Global & 129,6 & $1.549,3$ & 276,3 & 0,084 & $0,029-0,230$ & $1.419,7$ & 0,92 \\
\hline
\end{tabular}

(a) Densidad de incidencia por 100.000 personas/año; (b) No Expuestos para REGICOR (puntuación REGICOR $<5$, grupo de riesgo bajo; (c) Expuestos para REGICOR (puntuación REGICOR $\geq 5$, grupos de riesgo moderado, alto y muy alto; (d) RAe: riesgo atribuible expuestos; (e) FAe: fracción atribuible en expuestos o porcentaje riesgo atribuible en expuestos.

\section{Tabla 5}

Casos y densidad de incidencia esperados y observados para la función REGICOR según género.

\begin{tabular}{|c|c|c|c|c|c|c|c|c|}
\hline \multirow{2}{*}{ Variables } & \multicolumn{4}{|c|}{ Casos } & \multicolumn{5}{c|}{ Densidad de incidencia } \\
\cline { 2 - 9 } & Esperados & Observados & $\mathrm{p}$ & Esperada & Observada & Razón & Ic95\% & $\mathrm{p}$ \\
\hline Mujeres (186) & 3 & 1 & 0,315 & 163,0 & 54,1 & 0,3 & $0,24-157,5$ & 0,37 \\
\hline Hombres (512) & 15 & 18 & 0,596 & 298,4 & 357,4 & 1,2 & $0,39-1,75$ & 0,61 \\
\hline Global (698) & 18 & 19 & 0,867 & 262,1 & 276,7 & 1,05 & $0,47-1,91$ & 0,87 \\
\hline Densidad de incidencia por 100.000 personas/año.
\end{tabular}


Puede observarse que no existieron diferencias significativas entre lo esperado $\mathrm{y}$ lo observado.

Por grupos de riesgo cardiovascular según Regicor, en el grupo de bajo riesgo el número de casos predicho fue de 14, mientras los casos observados fueron $8(\mathrm{p}<0,05)$. En el grupo de riesgo moderado, se predijeron 4 casos y se observó 1 sólo caso $(\mathrm{p}<0,05)$.
La densidad de incidencia de la enfermedad cardiovascular (CI y ACV) fue de 360,1 por cada 100.000 trabajadores y año, resultando 477,1 y 53,9 en hombres y mujeres, respectivamente.

Se realizó análisis de regresión de riesgos proporcionales de Cox (tabla 6) en casos incidentes de CI (REGICOR) y en el total de casos de CI y cerebrovascular (combinada). En ambas resultaron significativas la Edad, PAD y Tabaco.

\section{Tabla 6}

Regresión de riesgos proporcionales de Cox en casos incidentes de cardiopatía isquémica (REGICOR) y en el total de casos de cardiopatía isquémica y cerebrovascular (combinada).

\begin{tabular}{|c|c|c|c|c|c|c|}
\hline REGICOR & \multicolumn{3}{|c|}{ UNIVARIANTE } & \multicolumn{3}{c|}{ MULTIVARIANTE } \\
\hline Variables & HR & IC95\% & $\mathrm{p}$ & HR & IC95\% & $\mathrm{p}$ \\
\hline Edad años & 1,19 & 1,$094 ; 1,301$ & $<0,001$ & 1,158 & 1,$059 ; 1,267$ & 0,001 \\
\hline Glucosa mg/dl & 1,02 & 1,$007 ; 1,033$ & 0,002 & 1,009 & 0,$994 ; 1,025$ & 0,25 \\
\hline Triglicéridos mg/dl & 1,00 & 1,$001 ; 1,008$ & 0,005 & 1,058 & 0,$997 ; 1,007$ & 0,45 \\
\hline PAS mm Hg & 1,05 & 1,$024 ; 1,075$ & $<0,001$ & 1,004 & 0,$960 ; 1,050$ & 0,86 \\
\hline PAD mm Hg & 1,09 & 1,$045 ; 1,136$ & $<0,001$ & 1,064 & 1,$018 ; 1,112$ & 0,006 \\
\hline DPP & 1,04 & 1,$002 ; 1,088$ & 0,039 & 1,004 & 0,$96 ; 1,054$ & 0,86 \\
\hline Colesterol total & 1,00 & 0,$994 ; 1,017$ & 0,36 & - & - & - \\
\hline cHDL & 0,99 & 0,$959 ; 1,032$ & 0,78 & - & - & - \\
\hline cLDL & 1,01 & 0,$997 ; 1,023$ & 0,15 & - & - & - \\
\hline cTotal/c-HDL & 1,13 & 0,$703 ; 1,810$ & 0,62 & - & - & - \\
\hline c-LDL/c-HDL & 1,37 & 0,$771 ; 2,451$ & 0,281 & - & - & - \\
\hline TG/c-HDL & 1,23 & 1,$041 ; 1,459$ & 0,015 & 1,09 & 0,$576 ; 2,062$ & 0,79 \\
\hline IMC & 1,08 & 0,$996 ; 1,171$ & 0,061 & - & - & - \\
\hline CC & 1,03 & 0,$993 ; 1,074$ & 0,103 & - & - & - \\
\hline ICT & 1,06 & 0,$917 ; 1,135$ & 0,06 & - & - & - \\
\hline PG\% & 1,02 & 0,$958 ; 1,093$ & 0,49 & - & - & - \\
\hline Formación & 0,90 & 0,$786 ; 1,030$ & 0,124 & - & - & - \\
\hline Sexo & 0,15 & 0,$020 ; 1,135$ & 0,07 & - & - & - \\
\hline Tabaco & 3,03 & 1,$193 ; 7,695$ & 0,02 & 3,497 & 1,$372 ; 8,909$ & 0,006 \\
\hline SMet & 2,38 & 0,$906 ; 6,269$ & 0,08 & - & - & - \\
\hline AFB & 3,40 & 0,$991 ; 11,668$ & 0,05 & 0,605 & 0,$162 ; 2,264$ & 0,45 \\
\hline PDL & & - & - & - \\
\hline
\end{tabular}

PAS: presión arterial sistólica; PAD: presión arterial diastólica; DPP: diferencia presión de pulso; cHDL: colesterol unido a lipoproteínas de alta densidad; cLDL: colesterol unido a lipoproteínas de baja densidad; IMC: índice de masa corporal; CC: circunferencia abdominal; ICT: índice cintura/talla; PG\%: porcentaje de peso graso; Formación: años de formación; SMet: Síndrome metabólico según criterios de la definición armonizada; Actividad física baja: según cuestionario internacional actividad física IPAQ. 
Tabla 6 (continuación)

Regresión de riesgos proporcionales de Cox en casos incidentes de cardiopatía isquémica (REGICOR) y en el total de casos de cardiopatía isquémica y cerebrovascular (combinada).

\begin{tabular}{|c|c|c|c|c|c|c|}
\hline COMBINADA & \multicolumn{3}{|c|}{ UNIVARIANTE } & \multicolumn{3}{c|}{ MULTIVARIANTE } \\
\hline Variables & HR & IC95\% & $\mathrm{p}$ & $\mathrm{HR}$ & IC95\% & $\mathrm{p}$ \\
\hline Edad años & 1,21 & 1,$120 ; 1,307$ & $<0,001$ & 1,174 & 1,$084 ; 1,272$ & $<0,001$ \\
\hline Glucosa mg/dl & 1,02 & 1,$005 ; 1,030$ & 0,007 & 1,011 & 0,$994 ; 1,028$ & 0,20 \\
\hline Triglicéridos mg/dl & 1,00 & 1,$001 ; 1,007$ & 0,005 & 1,003 & 0,$998 ; 1,008$ & 0,29 \\
\hline PAS mm Hg & 1,05 & 1,$031 ; 1,074$ & $<0,001$ & 1,007 & 0,$879 ; 1,115$ & 0,92 \\
\hline PAD mm Hg & 1,09 & 1,$051 ; 1,130$ & $<0,001$ & 1,062 & 1,$022 ; 1,103$ & 0,002 \\
\hline DPP & 1,04 & 1,$015 ; 1,076$ & 0,003 & 1,019 & 0,$980 ; 1,060$ & 0,34 \\
\hline Colesterol total & 1,00 & 0,$994 ; 1,015$ & 0,41 & - & - & - \\
\hline C-HDL & 0,99 & 0,$960 ; 1,024$ & 0,62 & - & - & - \\
\hline c-LDL & 1,01 & 0,$997 ; 1,020$ & 0,16 & - & - & - \\
\hline cTotal/c-HDL & 1,01 & 0,$859 ; 1,178$ & 0,94 & - & - & - \\
\hline IMC & 1,05 & 0,$970 ; 1,139$ & 0,22 & - & - & - \\
\hline CC & 1,03 & 0,$995 ; 1,065$ & 0,09 & - & - & - \\
\hline ICT & 1,06 & 1,$001 ; 1,122$ & 0,045 & 0,032 & 0,$001 ; 43,420$ & 0,35 \\
\hline PG\% & 1,00 & 0,$940 ; 1067$ & 0,96 & - & - & - \\
\hline Formación & 0,90 & 0,$796 ; 1,009$ & 0,07 & - & - & - \\
\hline Sexo & 0,11 & 0,$115 ; 0,841$ & 0,03 & 0,135 & 0,$018 ; 1,001$ & 0,05 \\
\hline Tabaco & 2,65 & 1,$191 ; 5,901$ & 0,02 & 3,059 & 1,$371 ; 6,828$ & 0,006 \\
\hline Smet & 0,48 & 0,$201 ; 1,155$ & 0,10 & - & - & - \\
\hline AC Física & 3,34 & 1,$147 ; 9,734$ & 0,03 & - & - & - \\
\hline Parly & & - & - & - \\
\hline
\end{tabular}

PAS: presión arterial sistólica; PAD: presión arterial diastólica; DPP: diferencia presión de pulso; cHDL: colesterol unido a lipoproteínas de alta densidad; cLDL: colesterol unido a lipoproteínas de baja densidad; IMC: índice de masa corporal; CC: circunferencia abdominal; ICT: índice cintura/talla; PG\%: porcentaje de peso graso; Formación: años de formación; SMet: Síndrome metabólico según criterios de la definición armonizada; Actividad física baja: según cuestionario internacional actividad física IPAQ.

\section{DISCUSIÓN}

Los estudios en la población laboral española sobre prevalencia de FRCV y SMet, valoración del riesgo cardiovascular mediante funciones validadas para España (REGICOR) y longitudinales para el conocimiento de la incidencia de patología cardiovascular, no son frecuentes $^{(25)}$.
La comparación de prevalencia de los distintos FRCV presenta la debilidad de las diferentes edades medias de las poblaciones estudiadas, así como el ámbito, laboral o poblacional, donde se realizan.

La prevalencia de obesidad, diabetes e hipertensión observada en la población de nuestro estudio es inferior a la publicada por Baena-Díez ${ }^{(8)}$, 
Comín ${ }^{(14)}$ y Medrano ${ }^{(15)}$. En cuanto al hábito de fumar, los hombres presentan prevalencia inferior a la de la cohorte de Manresa ${ }^{(13)}(60 \%)$ y Sánchez Chaparro $^{(26)}(51,3 \%)$, mientras que en mujeres la prevalencia observada por nosotros $(39,3 \%)$ es superior a la publicada por Sánchez Chaparro ${ }^{(26)}$, Baena-Díez $^{(8)}$, Comín ${ }^{(14)}$, Medrano ${ }^{(15)}$ y la ENS$2003^{(27)}$. Es posible que el tabaquismo femenino de nuestra muestra se encuentre vinculado a la combinación de baja edad media y ámbito de trabajo.

El nivel de actividad física no suele recogerse en los estudios, y cuando se hace lo es con indicadores no homogéneos. Frente al 61\% de sedentarismo según la ENS-2003(27), nuestro estudio obtiene una media del 50,3\%.

Los primeros estudios nacionales sobre síndrome metabólico, definido hace casi 30 años por Reaven $\mathrm{GM}^{(28)}$, son los de Álvarez EE et $\mathrm{al}^{(29)}$ y Lorenzo $\mathrm{C}$ et $\mathrm{al}^{(30)}$, con una prevalencia próxima al $25 \%$. Nuestro estudio, con un $18,9 \%$ en hombres y un $9,7 \%$ en mujeres, presenta una prevalencia similar a la dada por Alegría et al ${ }^{(31)}$ para el estudio MESYAS. Tanto en el análisis multivariante como en la determinación de la curva ROC, el SMet no figura como elemento asociado independientemente a la incidencia cardiovascular en estudios a diez años, con lo que, siguiendo a Fernández-Bergés et $\mathrm{al}^{(32)}$, se mantiene la controversia acerca de la aplicación clínica del diagnóstico de SMet. Es posible que el Smet precise mayor tiempo de evolución para repercutir en la incidencia cardiovascular, por lo que sería conveniente su valoración en cohortes de quince a veinte años de seguimiento.

En el análisis de riesgos proporcionales de Cox, tanto para los incidentes de primer episodio de CI como para cualquier episodio cardiovascular, tanto la edad como la tensión arterial y el tabaquismo son las variables independientemente asociadas con riesgo significativo de desarrollar dichos eventos. El tabaquismo presenta un riesgo significativo de 3,5 en CI y de 2,98 en CI y ACV combinados. Esta asociación pone en primer lugar las intervenciones para la promoción de la vida saludable y la deshabituación tabáquica para el control de la patología cardiovascular, seguida del control de la hipertensión, coincidiendo en ello con $\mathrm{Grau}^{(16)} \mathrm{y}$ Piniés et $\mathrm{al}^{(33)}$.

Royo-Bordonada et $\mathrm{al}^{(34)}$ insisten en la importancia de valorar la intervención clínica en función del riesgo cardiovascular, aun reconociendo que no hay evidencia suficiente que la anteponga al tratamiento individual de los factores de riesgo. La función adaptada de REGICOR es, según Comín et al ${ }^{(14)}$, la opción aplicable que muestra el mejor equilibrio en la capacidad de clasificación de riesgo de acontecimientos cardiovasculares, y según Marrugat et $\mathrm{al}^{(35)}$ predice con precisión y exactitud los acontecimientos coronarios a 10 años.

Salvo para Marrugat et al ${ }^{(35)}$, el riesgo de presentar un primer episodio de CI, mortal o no, obtenido en nuestro estudio es similar al observado por Vicente-Herrerro ${ }^{(36)}$, López-González ${ }^{(37)}$, Sánchez-Chaparro ${ }^{(26)}$ y Tauler ${ }^{(38)}$, éste último en lo referente a la proporción de trabajadores con REGICOR alto. Puede concluirse que el riesgo medio en la población laboral de CI, fatal o no, a diez años es bajo (inferior al 5\%).

La concordancia entre los casos esperados según la función REGICOR y los no esperados, permite aceptar que, de manera global, la capacidad predictiva de dicha función es adecuada. No alcanza el mismo nivel de concordancia según los grupos de riesgo, con predicción al alza en el grupo de bajo riesgo y a la baja en el moderado, en el que más casos. La ausencia de diferencias significativas entre las densidades de incidencia (DI) esperadas y observadas para ambas funciones colaboran en dicha afirmación. Estudios como los 
de Hermida-Ameijeiras ${ }^{(39)}$ y Jiméne ${ }^{(40)}$ indican que REGICOR subclasifica el grupo de riesgo moderado.

Si para Marrugat et al ${ }^{(35)}$ la mayoría de los casos coronarios se dan en sujetos con riesgo entre el $5 \mathrm{y}$ el $15 \%$, en nuestra población el $58 \%$ se dio en el grupo de riesgo medio, cuando se esperaba el $22 \%$, siendo resultados similares a los de Comín et al ${ }^{(14)}$.

Es posible que los valores de corte utilizados para clasificar como sujetos con bajo riesgo según REGICOR precisen modificación a la baja. Otra hipótesis para explicar la baja predicción según grupos de riesgo es que estas funciones subclasifiquen el nivel de riesgo al no atender a las variantes genéticas asociadas con CI, independientemente de los FRCV ${ }^{(41,42)}$. Persiste la necesidad de dotar a estas funciones de mayor valor predictivo según grupos de riesgo, por lo que nuevos estudios de incidencia cardiovascular con valoración del riesgo e inclusión de variantes genéticas aportarían mayores niveles de evidencia.

Encontramos en hombres una DI de CI, fatal o no, de 357,4 por cada 100.000 personas-año, inferior a las obtenida en la cohorte de trabajadores de Manresa ${ }^{(5)}$ (499,1 por cada 100.000), en parte explicable al presentar ésta una mayor tasa de tabaquismo. Igualmente, es inferior a la observada en recientes estudios sobre cohortes de tipo poblacional ${ }^{(8,14)}$. Aunque con diferentes magnitudes de asociación ${ }^{(14,15,16,43)}$, encontramos la persistencia de FRCV controlables como el tabaquismo y la hipertensión.

Del estudio realizado se concluye que la densidad de incidencia para cardiopatía isquémica, fatal o no, resultó de 276,3 por cada 100.000 personas-año, mientras que la de cardiopatía isquémica y accidente cerebrovascular combinadas fue de 360,1 por cada 100.000 personas-año, inferiores a las obtenidas en otras cohortes laborales. La función REGICOR valora adecuadamente el riesgo cardiovascular de manera global, perdiendo capacidad predictiva según grupos de riesgo. Además, el tabaquismo y la presión arterial se muestran como los factores de riesgo cardiovascular modificables con mayor asociación o riesgo proporcional para la aparición de una enfermedad cardiovascular.

Las limitaciones del presente estudio se consideran derivadas de la extracción de la población laboral en la que se realizó el estudio, la perteneciente a la Administración Pública (formación, clase social ocupacional...). Así, advertimos la presencia de menos mujeres que hombres en la muestra, con una tasa de participación del $66,9 \%$ que, aunque próxima, no alcanza el 70\% establecido como mínimo. Para la evaluación de la incidencia de evento duro fatal se precisan cohortes más equilibradas por sexo, así como de mayor tamaño.

\section{BIBLIOGRAFÍA}

1. Instituto Nacional de Estadística. Ministerio Economía y Empresa. España. (Internet).Disponible desde: http://www. ine.es/prensa/edcm_2017.pdf. Consultado el 24/01/2019.

2. Dégano IR, Salomaa V, Veronesi G, Ferrieres J, Kirchberger I, Laks T et al.

The Acute Myocardial Infarction Trends in Europe (AMITIE) Study Investigators. Twenty-five-year trends in myocardial infarction attack and mortality rates, and case-fatality, in six European populations. Heart. 2015 Sep;101(17):1413-21. doi: 10.1136/heartjnl-2014-307310. Epub 2015 Apr 8.

3. Sekikawa A, Miyamoto Y, Miura K, Nishimura K, Willcox BJ, Masaki KH et al. Continuous decline in mortality from coronary heart disease in Japan despite a continuous and marked rise in total cholesterol: Japanese experience after the Seven Countries Study. J Epidemiol. 2015 Oct; 44(5):1614-24. doi: 10.1093/ije/dyv143. Epub 2015 Jul 16.

4. Instituto Nacional de Estadística. Ministerio Economía y Competitividad. España. (Internet). Disponible desde: 
http://www.ine.es/jaxiT3/Datos.htm?t=7947. Consultado el $19 / 02 / 2016$.

5. Gil M, Martí H, Elosúa R, Grau M, Sala J, Masiá R et al. Análisis de la tendencia en la letalidad, incidencia y mortalidad por infarto de miocardio en Girona entre 1990 y 1999. Rev Esp Cardiol. 2007;60 (4):349-56.

6. Medrano MJ, Boix R, Cerrato E, Ramírez M. Incidencia y prevalencia de cardiopatía isquémica y enfermedad cerebrovascular en España: revisión sistemática de la literatura. Rev Esp Salud Pública. 2006; En; 80 (1): 5-15.

7. López-SuárezA, Bascuñana A, Elvira-González J, BeltránRobles M, Boza-Lobatón A, Solís-Díaz R. Incidencia poblacional de enfermedad cardiovascular y mortalidad en los adultos de 50-75 año. Rev Clin Esp.2013; 213(6): 278-84.

8. Baena-Díez JM, Vidal-Solsona M, Byram AO, GonzálezCasafont I, Ledesma-Ulloa G, Martí-Sans N. Epidemiología de las enfermedades cardiovasculares en atención primaria. Estudio Cohorte Zona Franca de Barcelona. Rev Esp Cardiol. 2010; 63(11):1261-9

9. Vila-Córcoles A, Forcadell MJ, De Diego Cabanés, Ochoa- Gondar O, Rull B, Barnes L et al. Incidencia y mortalidad por infarto agudo de miocardio en la población mayor de 60 años del área de Tarragona. Rev Esp Salud Publica. 2015 noviembre-diciembre.

10. Félix-Redondo FJ, Lozano-Mera L, Álvarez-Palacio P, Grau-Magana M, Ramírez-Romero JM, Fernández-Bergés $\mathrm{D}$ et al. Impacto de los factores de riesgo cardiovascular en la población extremeña: aportación de la cohorte HERMEX para una estrategia preventiva. Aten Primaria. 2018. https:// doi.org/10.1016/j.aprim.2018.11.006.

11. Vila-Córcoles A, Satue-Gracia E, Ochoa-Gondar O, De Diego-Cabanés C, Vila-Rovira A, Blade J et al. Incidencia y letalidad del ictus isquémico en la población mayor de 60 anos del área de Tarragona, España (2008-2011). Rev Neurol 2014; 59: 490-6.

12. Vázquez-Oliva G, Zamora A, Ramos R, Marti R, Subirana I, Grau M et al. Acute Myocardial Infarction
Population Incidence and Mortality Rates, and 28-day Casefatality in Older Adults. The REGICOR Study. Rev Esp Cardiol (Engl Ed). 2018 Sep;71(9):718-725. doi: 10.1016/j. rec.2017.10.019. Epub 2017 Nov 22.

13. Tomàs L, Varas C, Pérez I, Puig T, Balaguer I. Factores de riesgo y morbimortalidad coronaria en una cohorte laboral mediterránea seguida durante 28 años. Estudio de Manresa. Rev Esp Cardiol. 2001 Oc; 54 (10): 1146-1154.

14. Comín E, Solanas P, Cabezas C, Subirana I, Ramos R, Gené-Badía J et al. Rendimiento de la estimación del riesgo cardiovascular en España mediante la utilización de distintas funciones. Rev Esp Cardiol. 2007;60(7):693-702.

15. Medrano MJ, Pastor-Barriuso R, Boix R, Barrio J, Damián J, Álvarez R et al. Riesgo coronario atribuible a los factores de riesgo cardiovascular en población española. Rev Esp Cardiol. 2007; 60 (12):1250-6

16. Grau M, Subirana I, Elosua R, Fitó M, Covas MI, Sala $\mathrm{J}$ et al. REGICOR investigators. Why should population attributable fractions be periodically recalculated? $\square$ : An example from cardiovascular risk estimation in southern Europe. Preventive Medicine. Volume 51, Issue 1, July 2010, Pages 78-84

17. Grupo Dieta y riesgo cardiovascular en España (DRECE II). Descripción de la evolución del perfil cardiovascular. Med Clin. 2000;115:726-9.

18. Alcohol y atención primaria de la salud: informaciones clínicas básicas para la identificación y el manejo de riesgos y problemas. (internet). Disponible en: http://www. who.int/substance_abuse/publications/alcohol_atencion primaria.pdf. Consultado el 19/01/2016.

19. Román-Viñas B, Serra-Majem L, Hagströmer M, Segura-Cardona R, Ribas-Barba L. International Physical Activity Questionnaire: Reliability and Validity in Spain. Med Sci Sports Exerc, 38 (2006), p. S563.

20. Alberti KGMM, Eckel RH, Grundy SM, Zimmet PZ, Cleeman JI, Donato KA et al. Harmonizing the metabolic syndrome. A Joint Interm Statement of the International 
Diabetes Federation Task Force on Epidemiology and Prevention; National Heart, Lung and Blood Institute; American Heart Association; World. Heart Federation; International Atherosclerosis Society; and International Association for the Study of Obesity. Circulation. 2009; $120: 1640-5$

21. Marrugat J, Subirana I, Comín E, Cabezas C, Vila $\mathrm{J}$, Elosúa $\mathrm{R}$ et al. Validity of an adaptation of the framingham cardiovascular risk function: the VERIFICA study. J Epidemiol Community Health. 2007; 61:40-47. Disponible en internet http://www.regicor.org/media/upload/ research/pdf/171830141334152662.pdf. Consultado el día $17 / 12 / 2015$

22. Sans S, Fitzgerald A, Royo D, Conroy R, Graham I. Calibración de la tabla SCORE de riesgo cardiovascular para España. Rev Esp Cardiol. 2007; 60(5):476-85

23. ISAK. International standards for anthropometric assessment. Unerdale: ISAK; 2001.

24. Mancia G, Fagard F, Narkiewicz K, Redon J, Zanchetti A, Böhm M et al. Grupo trabajo para el manejo de la hipertensión arterial de la Sociedad Europea de Hipertensión (ESH) y la Sociedad Europea de Cardiología. Guía de práctica clínica de la ESH/ESC para el manejo de la hipertensión Arterial. 2013. Hipertens riesgo vasc. 2013;30(Supl 3):4-91.

25. Maiques A, Brotons C, Villar F et al. Grupo de Prevención Cardiovascular del PAPPS. Aten Primaria. 2014 Jun; 46 Suppl 4:3-15. doi: 10.1016/S0212-6567(14)70047.

26. Sánchez-Chaparro MA, Román-García JR, CalvoBonacho E, Gómez-Larios T, Fernández-Meseguer A, SáinzGutiérrez JC et al. Prevalencia de factores de riesgo vascular en la población laboral española. Rev Esp Cardiol. 2006; 59 (5) 421-30.

27. Encuesta Nacional de Salud 2003. Internet. Disponible en: http://www.msssi.gob.es/estadEstudios/estadisticas/encuestaNacional/home.htm. Consultado el 02/02/2015

28. Reaven GM. Role of insulin resistance in human disease. Diabetes. 1988;37:1595-607.
29. Alvarez EE, Ribas L, Serra L. Prevalencia del síndrome metabólico en la población de la Comunidad Canaria. Med Clin (Barc). 2003;120:172-4.

30. Lorenzo C, Serrano-Ríos M, Martínez-Larrad MT, Gabriel R, Williams K, Gómez-Gerique JA et al. Central adiposity detemines prevalence difference of the metabolic syndrome. Ob Res. 2003;11:1480-7.

31. Alegría E, Cordero A, Grima A, Laclaustra M, Grima A, León $\mathrm{M}$ et al. Prevalencia del síndrome metabólico en población laboral española: Registro MESYAS. Rev Esp Cardiol. 2005;58:797-806.

32. Fernández-Bergés D, Cabrera A, Sanz H, Elosua R, Guembe MJ, Alzamora M et al. Síndrome metabólico en España: prevalencia y riesgo coronario asociado a la definición armonizada y a la propuesta por la OMS. Estudio DARIOS. Rev Esp Cardiol. 2012; 65(3):241-248.

33. Piniés JA, González-Carril F, Arteagoitia JM, Irigoien I, Altzibar JM, Rodríguez-Murua JL et al. Development of a prediction model for fatal and non-fatal coronary heart disease and cardiovascular disease in patients with newly diagnosed type 2 diabetes mellitus: the Basque Country Prospective Complications and Mortality Study risk engine (BASCORE). Diabetologia. 2014 Nov; 57 (11): 2324-33. doi: 10.1007 / s00125-014-3370-1. Epub 2014 Sep 1.

34. Royo-Bordonada MA, Lobos JM, Villar F, Sans S, Pérez A, Pedro-Botet $\mathrm{J}$ et al. Comentarios del Comité Español Interdisciplinario de Prevención Cardiovascular (CEIPC) a las Guías Europeas de Prevención Cardiovascular 2012. Clin Invest Arterioscl. 2013. http://dx.doi.org/10.1016/j.arteri.2013.03.003. Consultado el día 9/06/2015.

35. Marrugat J, Vila J, Baena-Díez JM, Grau M, Sala J, Ramos $\mathrm{R}$ et al. Validez relativa de la estimación del riesgo cardiovascular a 10 años en una cohorte poblacional del estudio REGICOR. Rev Esp Cardiol. 2011;64 (5):385-394.

36. Vicente-Herrero MT, López González AA, RamírezÍñiguez MV, Capdevila-García L, Terradillos-García MJ, Aguilar-Jiménez E. Parámetros de riesgo cardiovascular, síndrome metabólico y consumo de alcohol en población 
laboral. Endocrinol Nutr. 2015;62(4):161-167. Disponible en: http://dx.doi.org/10.1016/j.endonu.2015.01.002. Consultado el $22 / 12 / 2015$.

37. López-González AA, Bennasar-Veny M, Tauler P, Aguilo $\mathrm{P}$, Tomàs-Salvà $\mathrm{M}$, Yáñez $\mathrm{A}$ et al. Desigualdades socioeconómicas y diferencias según sexo y edad en los factores de riesgo cardiovascular. Gac Sanit. 2015;29 (1):27-36

38. Tauler P, Bennasar-Veny M, Morales-Asencio JM, Lopez-Gonzalez AA, Vicente-Herrero T, et al. (2014) Prevalence of Premorbid Metabolic Syndrome in Spanish Adult Workers Using IDF and ATPIII Diagnostic Criteria: Relationship with Cardiovascular Risk Factors. PLoS ONE 9(2): e89281.doi:10.1371. Consultado 22/12/2015

39. Hermida-Armejeiras A, López-Paz JE, Riveiro-Cruz MA, Calvo-Gómez C. Carotid intima-media thickness distribution according to the stratification of cardiovascular risk by means of Framingham-REGICOR and score function charts. Hipertens Riesgo Vasc. 2016 Apr-Jun;33(2):51-7
40. Jiménez M, Dorado L, Hernández-Pérez M, Alzamora MT, Pera G, Torán P et al. Ankle-brachial index in screening for asymptomatic carotid and intracranial atherosclerosis.. Atherosclerosis. 2014 Mar;233(1):72-5.

41. Lluís-Ganella C, Gavín L, Subirana I, Sentí M, JiménezConde J, Marrugat J et al. Efecto aditivo de diferentes variantes genéticas en el riesgo de cardiopatía isquémica. Rev Esp Cardiol. 2010;63(8):925-33

42. Lluis-Ganella C, Subirana I, Lucas G, Tomás M, Muñoz $\mathrm{D}$, Sentí $\mathrm{M}$ et al. Assessment of the value of a genetic risk score in improving the estimation of coronary risk. Atherosclerosis. 2012 Jun; 222(2): 456-463.

43. Graciani A, Zuluaga-Zuluaga MC, Banegas JR, LeónMuñoz LM, de la Cruz JJ, Rodríguez-Artalejo F. Mortalidad cardiovascular atribuible a la presión arterial elevada en la población española de 50 años o más. Med Clin Barc). 2008 Jun 28;131(4):125-9. 